\title{
Sonographic assessment of abdominal fat distribution in infancy
}

\author{
Susanne Holzhauer - Renate M. L. Zwijsen • Vincent W. V. Jaddoe · Guenther Boehm • \\ Henriette A. Moll · Paul G. Mulder • Veronica A. Kleyburg-Linkers · Albert Hofman • \\ Jacqueline C. M. Witteman
}

Received: 8 March 2009/ Accepted: 30 June 2009/Published online: 29 July 2009

(c) The Author(s) 2009. This article is published with open access at Springerlink.com

\begin{abstract}
There is growing evidence that not only the total amount of fat, but also the distribution of body fat determines risks for metabolic and cardiovascular disease. Developmental studies on factors influencing body fat distribution have been hampered by a lack of appropriate techniques for measuring intraabdominal fat in early life. Sonography, which is an established method for assessing abdominal fat distribution in adults, has not yet been evaluated in infants. To adapt the sonographic measurement of abdominal fat distribution to infants and study its reliability. The Generation $\mathrm{R}$ study, a population-based prospective cohort study. We included 212 one- and 227 two-year old Dutch infants in the present analysis. Sixty-two infants underwent replicate measurements to assess reproducibility. We developed a standardized protocol to measure the
\end{abstract}

S. Holzhauer · V. W. V. Jaddoe - V. A. Kleyburg-Linkers ·

A. Hofman - J. C. M. Witteman

The Generation R Study Group, Erasmus Medical Center,

Rotterdam, The Netherlands

e-mail: Susanne.holzhauer@charite.de

S. Holzhauer · V. W. V. Jaddoe · P. G. Mulder · A. Hofman J. C. M. Witteman ( $\triangle)$

Department of Epidemiology \& Biostatistics, Erasmus Medical Center, P.O. Box 2040, 3000 CA Rotterdam, The Netherlands

e-mail: j.witteman@erasmusmc.nl

R. M. L. Zwijsen

Numico Research, Wageningen, The Netherlands

V. W. V. Jaddoe · G. Boehm · H. A. Moll

Department of Pediatrics, Sophia Children's Hospital, Erasmus

Medical Center, Rotterdam, The Netherlands

G. Boehm

Numico Research, Friedrichsdorf, Germany thickness of (1) subcutaneous and (2) preperitoneal fat in the upper abdomen of infants. To this end we defined infancy specific measurement areas to quantify fat thickness. Reproducibility of fat measurements was good to excellent with intraclass correlation coefficients of $0.93-0.97$ for intra-observer agreement and of 0.89-0.95 for inter-observer agreement. We observed a pronounced increase in preperitoneal fat thickness in the second year of life while subcutaneous fat thickness increased only slightly, resulting in an altered body fat distribution. Gender did not significantly influence fat distribution in the first two years of life. Our age specific protocol for the sonographic measurement of central subcutaneous and preperitoneal fat is a reproducible method that can be instrumental for investigating fat distribution in early life.

Keywords Abdominal fat distribution - Body composition · Infancy · Intraabdominal fat · Sonography · Visceral fat

\section{Introduction}

The prevalence of obesity has been steadily increasing worldwide during the last decades [1]. Obesity is associated with metabolic disturbances, including insulin resistance and type II diabetes mellitus, as well as cardiovascular disease in adults [1]. Several studies have shown that visceral adiposity poses a higher risk for developing obesity related disorders than overall adiposity, suggesting the particular role of intraabdominal fat in metabolic and cardiovascular disease [2-5]. The mechanisms that account for the association between intraabdominal fat and disease as well as factors and critical time periods influencing the development of intraabdominal fat remain controversial. To develop 
prevention strategies, systematic investigations on fat development and factors influencing fat distribution throughout life are required. Research on fat distribution has been hampered by a lack of appropriate methods that can be applied to all age groups and as a consequence longitudinal studies on the development of intraabdominal fat throughout childhood have not yet been undertaken.

The most accurate and reproducible techniques for abdominal fat distribution in adults are a computed tomography CT scan [6], which necessitates radiation exposure, or abdominal magnetic resonance imaging MRI [7], which is cost-intensive and prone to movement artefacts in infants, because extended scanning times are required. Thus, both techniques are of limited use for pediatric investigations, especially when repeated measurements are involved.

Simpler methods for estimating abdominal adiposity as waist circumference, waist hip ratio or skinfold thickness do not directly quantify abdominal fat compartments or may be unrelated to visceral fat in infants or young children $[8,9]$.

Sonography is an easy accessible and non-invasive method, which is particularly useful for investigations in children. Different methods for estimating intraabdominal adipose tissue with sonography have been established in adults [10-12]. Suzuki et al. [12] estimated abdominal fat distribution by measuring preperitoneal fat thickness and relating it to subcutaneous fat thickness, both measured in the upper abdomen. The so called abdominal wall fat index has been shown to be strongly correlated with CT measurements of abdominal fat distribution in adults. Beyond, both preperitoneal fat thickness and abdominal wall fat index have been identified as risk factors for cardiovascular disease, insulin resistance or type II diabetes independent of generalized obesity in adults [11-16]. However, in children data on the sonographic estimation of abdominal fat compartments is sparse and restricted to older children [17].

The aim of the present study was (1) to adapt the sonographic assessment of abdominal fat distribution via measurement of preperitoneal and subcutaneous fat to infants, (2) to study reliability of this method, (3) and to characterize how the results of this measurement method relate to anthropometric measures as well as age and sex.

\section{Methods}

\section{Study population}

The study was embedded in the Generation R Study, a population-based prospective cohort study, designed to study growth, development and health from fetal life until young adulthood. Rationale and design of the Generation $\mathrm{R}$ study have been described elsewhere in detail [18, 19]. Eligible mothers were resident in Rotterdam, the Netherlands at their delivery date (April 2002 until January 2006). The total cohort consists of 9,778 pregnant women of different ethnicities. A sub-cohort of Dutch ethnicity and expected date of birth between March 2003 and June 2005 was selected for more detailed measurements (Generation R Focus Study). The study was approved by the Medical Ethical Committee of the Erasmus Medical Center, Rotterdam. Written informed consent was obtained from all participants. We included 439 Dutch infants who underwent an ultrasound examination of the abdomen at their regular 1 or 2 years visit between May 2006 and March 2007 in the actual analysis.

Data collection and measurements

Pregnancy and birth characteristics: Date of birth, birth weight, gestational age and gender were obtained from midwife and hospital registries. Breastfeeding information: Information on duration of breastfeeding was recorded in an interview at the time of the ultrasound investigation. The interviewer was blind with respect to the measurement outcome. Anthropometrics: Weight was measured in naked infants to the nearest grams by using an electronic scale $\left(\mathrm{SECA}^{\circledR}\right)$. Height was measured in infants in supine position to the nearest $0.1 \mathrm{~cm}$ by a neonanometer (Holtain Limited ${ }^{\circledR}$ ). Body mass index (BMI) was calculated as weight/height ${ }^{2}\left(\mathrm{~kg} / \mathrm{m}^{2}\right)$.

Sonographic assessment of abdominal subcutaneous and preperitoneal fat

All ultrasound examinations of the study were performed with an ATL HDI 5000 (WA, Bothell). Preperitoneal and subcutaneous fat thickness were measured with a linear (L12-5 MHz) transducer according to the method described by Suzuki et al. in adults [12]. The infants were in a supine position. A linear transducer was placed perpendicular to the skin surface on the median upper abdomen. Great care was taken not to apply any pressure on the abdominal wall in order to avoid compression the tissue layers. We scanned longitudinally from the xiphoid process to the navel along the midline (linea alba). We took three ultrasound pictures just below the xiphoid process. The change in slope of the different layers to-as much as possible_-parallel layers served as the apical reference point to measure (Fig. 1). All measurements were performed off-line. Preperitoneal fat was measured as 1. Distance of the linea alba to the peritoneum on top of the liver (PP-distance) and 2. Areas of 1 and $2 \mathrm{~cm}$ length along the midline, starting from the reference point in 


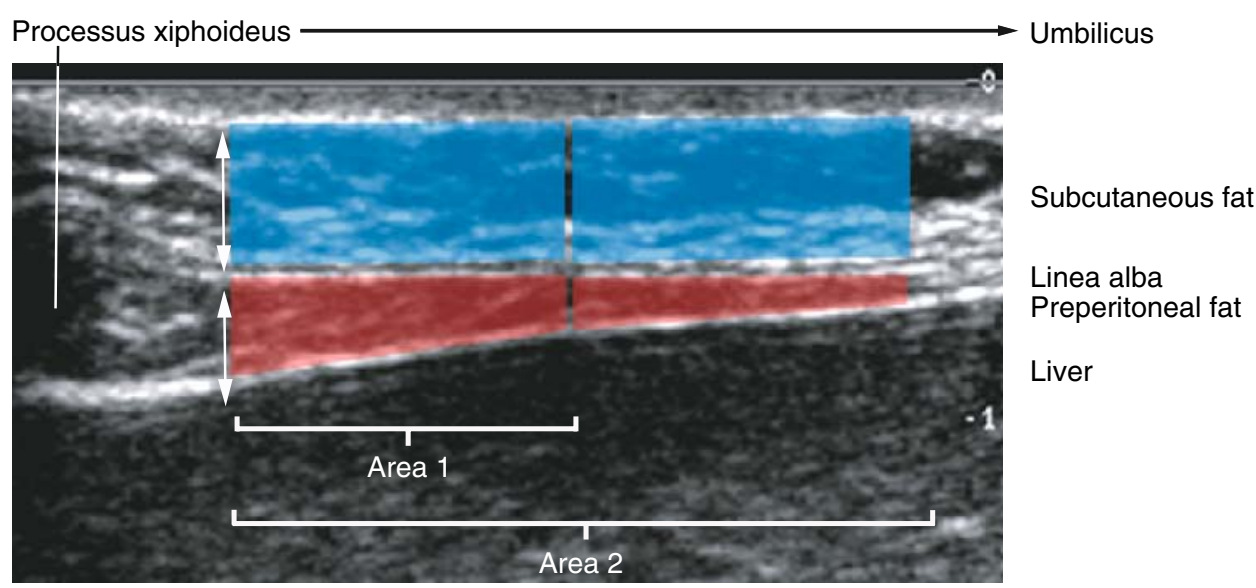

Fig. 1 Measurements of subcutaneous and preperitoneal fat. Arrows indicate subcutaneous (SC) and preperitoneal (PP) distance. Blue fields indicate SC area 1 and 2 measurements; red fields indicate PP area 1 and 2 measurements. 1 and 2 refer to the length of the area measurements with $1=1 \mathrm{~cm}, 2=2 \mathrm{~cm}$. Characteristic ultrasound image of abdominal fat layers, longitudinal midline cut in height of the upper abdomen (Color figure online) direction of the navel (PP-area-1, PP-area-2). Subcutaneous fat was measured as 1 . Distance of the inner surface of subcutaneous tissue to the linea alba (SC-distance) and 2. Areas of 1 and $2 \mathrm{~cm}$ length along the midline starting from the reference point in direction of the navel (SCarea-1, SC-area-2). Ratios of preperitoneal fat and subcutaneous fat were measured as (a) PP/SC-distance, (b) PP/SC-area-1 and (c) PP/SC-area-2. All pictures were taken when children were relaxed and showed no or little movements. As the breathing phase and breathing intensity could not be completely standardized in infants of that young age pictures were taken when tissue layers were as much as possible parallel in preperitoneal measurements (end of inspiration). We chose the optimal image for measurements according to plane of section closest to midline cut and, again, the parallelism of the fat layers. All pictures were taken by two well trained examiners $(\mathrm{SH}, \mathrm{VK})$.

\section{Reproducibility}

To assess intra-observer agreement one single examiner (SH) took all pictures twice in a single patient as described above. Pictures were taken at the same occasion; all measurements were performed off-line with a minimum time interval of 1 week in between measurements-and blind for the results of the respective first measurements-by the same investigator $(\mathrm{SH})$.

To assess inter-observer agreement two examiners $(\mathrm{SH}$, VK) took pictures at the same occasion, but blind for the results of the other examiner. All off-line measurements were performed by the same examiner ( $\mathrm{SH}$ ) with a minimum interval time of 1 week between measurements.
Statistical analysis

Relations between variables were assessed by correlations (Spearman's rho). As the distributions of all measures of fat thickness as well as the derived ratios were skewed we $\log$ transformed them to obtain normal distributions for analysis. We also computed age and gender adjusted SD scores for BMI based on Dutch reference values [20, 21]. To assess the effect of sex and age on measures of fat thickness independent of differences in height and weight we compared geometric means with analysis of covariance. Intra- and inter-observer agreements were examined using intraclass correlation coefficients (ICC) and their 95\% confidence intervals. An ICC of 1 indicates that all of the observed variation is caused by between subject variations. A $p$-value of less than 0.05 was regarded as significant. All data were analyzed using the SPSS statistical package (SPSS Inc. ${ }^{\circledR}$, Chicago, IL Version 15 for Windows).

\section{Results}

Characteristics of the study population

Maternal, neonatal and postnatal characteristics of the 439 individuals included in the analysis are given in Table 1. BMI SD scores-an age and sex adjusted measure of obesity-did not differ significantly between 1 and 2 years old infants in our study population. Compared to the Dutch national reference values infants in this study had lower BMI (BMI SD score in 1 year old infants -0.2 SD 0.9, in 2 years old infants -0.3 SD 1.0) [20,21]. Only $7 \%$ of 1 year and $11 \%$ of 2 year-old infants of our study 
Table 1 Subject characteristics as percentage. Continuous values are presented as mean and standard deviation (SD)

\begin{tabular}{|c|c|c|c|c|c|}
\hline & \multicolumn{2}{|c|}{1 year $(n=212)$} & \multicolumn{2}{|c|}{2 years $(n=227)$} & \multirow[t]{2}{*}{$p$-value } \\
\hline & Mean & SD & Mean & SD & \\
\hline \multicolumn{6}{|l|}{ Characteristics mother } \\
\hline Age (years) & 32.0 & 3.8 & 32.0 & 3.9 & 0.79 \\
\hline Pre pregnant body mass index $\left(\mathrm{kg} / \mathrm{m}^{2}\right)$ & 23.2 & 3.6 & 23.6 & 4.4 & 0.32 \\
\hline Duration of breastfeeding (months) & 3.0 & 2.3 & 2.6 & 2.8 & 0.64 \\
\hline Parity (\% nulliparous) & 60.4 & & 65.6 & & 0.58 \\
\hline \multicolumn{6}{|l|}{ Characteristics child } \\
\hline Gender ( $\%$ male $)$ & 49.1 & & 52.9 & & 0.43 \\
\hline Gestational age (weeks) & 40.0 & 1.7 & 40.3 & 1.4 & 0.05 \\
\hline Birth weight $(\mathrm{g})$ & 3,487 & 533 & 3,528 & 526 & 0.42 \\
\hline Age (months) & 13.7 & 2.3 & 25.3 & 1.3 & $<0.001$ \\
\hline Current weight $(\mathrm{kg})$ & 9,968 & 1,410 & 12,442 & 1,891 & $<0.001$ \\
\hline Current length $(\mathrm{cm})$ & 78.1 & 3.8 & 89.1 & 3.2 & $<0.001$ \\
\hline Current BMI $\left(\mathrm{kg} / \mathrm{m}^{2}\right)$ & 16.5 & 1.1 & 15.5 & 2.6 & $<0.001$ \\
\hline Current BMI SD score & -0.2 & 0.9 & -0.3 & 1.0 & 0.27 \\
\hline
\end{tabular}

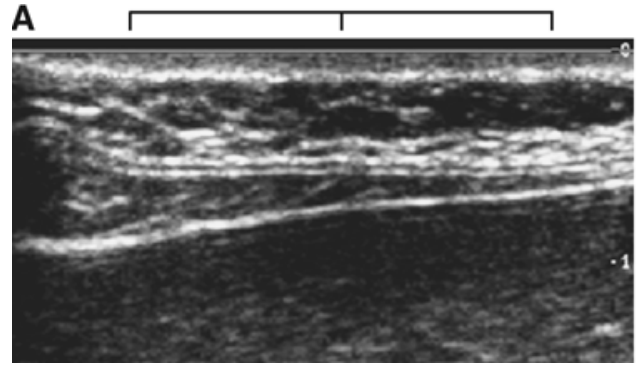

Fig. 2 Anatomy of fat layers in 1 and 2 year old infants. Characteristic ultrasound images that demonstrate the variation of the anatomic structures of fat layers within the study population. Longitudinal midline cut in height of the upper abdomen. a Immature

population were classified as at risk for overweight or overweight, defined as at or above the 85th percentile for age and sex of the Dutch reference values [22]. Infants of 1 and 2 years did not differ with regard to maternal BMI, duration of breastfeeding or parity, all factors that may influence body composition and fat distribution.

Sonographic assessment of preperitoneal and subcutaneous fat

The anatomy of preperitoneal fat in 1 and 2 years old infants showed a significant inter-subject variation and differed from the layout of these structures in adults (Fig. 2). In particular preperitoneal fat layers were extremely thin in some of the infants. To optimize precision and accuracy of our technique and to allow for age specific differences in anatomy, we therefore not only measured the thickness as distance between upper and lower border of the respective fat layer, but introduced areas of preperitoneal and subcutaneous fat (Fig. 1) as a measure of thickness of fat layers.

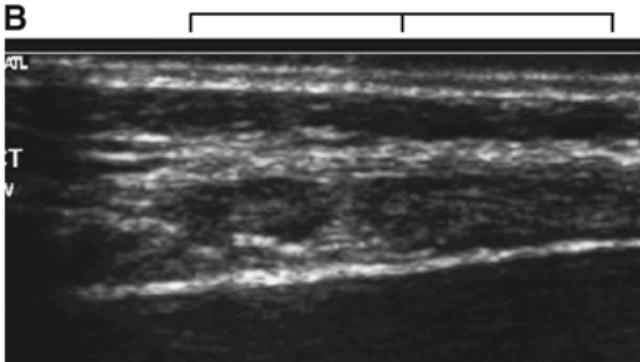

structure in an 11 months old infant, b Mature structure in a 26 months old infant. Brackets indicate 1 and $2 \mathrm{~cm}$ length, respectively

Performance of the technique

Correlations between measures of preperitoneal and subcutaneous fat

Measurements of (1) distance, (2) area of $1 \mathrm{~cm}$ length (area 1) and (3) area of $2 \mathrm{~cm}$ length (area 2) as alternative measurements to assess preperitoneal and subcutaneous fat thickness, were highly correlated among each other (Spearman's rho PP-area-1/PP-distance $r=0.86$, PP-area2/PP-distance $r=0.81$, PP-area-1/PP-area-2 $r=0.97$, SCarea-1/SC-distance $r=0.93$, SC-area-2/SC-distance $r=$ 0.92 , SC area 1SC-area-1/SC-area-2 $r=0.99$ ). Age or sex did not substantially influence the strength of the respective associations.

Intra- and inter-observer agreement of measures of abdominal fat thickness

Table 2 summarizes ICC on intra- and inter-observer agreement for all measurements of fat and their respective 
Table 2 Intra- and interobserver agreement of different preperitoneal and subcutaneous measurements

\begin{tabular}{lll}
\hline & $\begin{array}{l}\text { Intraobserver agreement } \\
\text { ICC }(\text { CI 95\%) }\end{array}$ & $\begin{array}{l}\text { Interobserver agreement } \\
\text { ICC }(\text { CI 95\% })\end{array}$ \\
\hline PP-area-1 & $0.93(0.86,0.97)$ & $0.95(0.90,0.97)$ \\
SC-area-1 & $0.97(0.93,0.99)$ & $0.96(0.92,0.98)$ \\
PP-area-2 & $0.96(0.91,0.98)$ & $0.93(0.87,0.97)$ \\
SC-area-2 & $0.97(0.93,0.99)$ & $0.95(0.91,0.97)$ \\
SC-distance & $0.97(0.94,0.99)$ & $0.94(0.88,0.97)$ \\
PP-distance & $0.89(0.77,0.95)$ & $0.91(0.84,0.95)$ \\
SC-distance & $0.95(0.90,0.98)$ & $0.90(0.82,0.95)$ \\
\hline
\end{tabular}

Data of intraobserver agreement $(n=26)$ and interobserver agreement $(n=36)$ are presented as intraclass correlation coefficients (ICC) and corresponding 95\% confidence intervals (CI 95\%)

${ }^{a}$ Interobserver agreement refers to the sonographic examination including taking ultrasound images; measurements were taken off line by one single observer with a minimum of 1 week time interval between measurements. $P P$ preperitoneal, $S C$ subcutaneous

derived ratios. Intra-observer agreement was good to excellent for all measures of preperitoneal and subcutaneous fat with ICC ranging from 0.89 (PP-distance) to 0.97 (SC-area-1, SC-area-2). Inter-observer agreement, which in the current study only refers to taking the ultrasound images, showed comparable results with ICC ranging from 0.90 (SC-distance) to 0.96 (SC-area-1). Area measurements had a slightly better reproducibility than measures of distance. Investigations were particularly difficult in 1 year old infants due to the relatively thin fat layers and frequent non compliance/restlessness of infants. We therefore also investigated agreement exclusively in the 14 months old infants. Here intra- and interobserver agreement were lower compared to the whole group with ICC ranging from 0.78 (PP-area-2) to 0.97 (SC-area-2).

Correlations between measures of preperitoneal and subcutaneous fat

Measures of preperitoneal fat were only weakly correlated with corresponding measures of subcutaneous fat (Spearman's rho PP area, SC area in 1 year old infants $r=0.31$, 2 years $r=0.38, P<0.001)$ indicating individual variation in abdominal fat distribution. In line with this finding, the distribution of ratios of preperitoneal to subcutaneous fat was broad (Ratio PP/SC-area-2 range 0.17-2.43). Strength of association was not substantially altered by age group or sex. All results for PP-area-2 and SC-area-2 were comparable to the respective area 1 and distance measures of preperitoneal and subcutaneous fat thickness (data not shown).
Correlation of measures of preperitoneal and subcutaneous fat with BMI

To compare the sonographic measurements of abdominal fat compartments with BMI-an established parameter for the assessment obesity-we assessed correlations between BMI and sonographic measurements. As shown in Fig. 3, BMI was only moderately correlated with measures of subcutaneous fat thickness (Spearman's rank correlation BMI, SC-area-2 $r=0.41, P<0.01)$. We did not find a correlation between BMI and measures of preperitoneal fat (Spearman's rank correlation BMI, PP-area-2 $r=-0.012$ ). As a consequence BMI was inversely related to measures of abdominal fat distribution as assessed by ratio PP-area-2/ SC-area- $r=-0.36, P<0.01)$. Thus, the higher the BMI the more the fat distribution changed towards subcutaneous abdominal fat. Results were comparable in 1 and 2 years old infants and between sexes. All results for PP-area-2 and SC-area-2 were comparable to the other measures of preperitoneal and subcutaneous fat thickness (data not shown).

\section{Effect of sex}

It is well established in adults that sex influences fat distribution with males being more prone to intraabdominal fat accumulation. We therefore examined the influence of sex on thickness of preperitoneal and subcutaneous fat as well as on abdominal fat distribution at the age of 1 and 2 years, respectively. Girls had more subcutaneous fat than boys; the preperitoneal to subcutaneous fat ratio did not differ significantly between boys and girls (geometric mean (CI95\%) for PP-area-2: $29.7(28.2 ; 31.3)$ in males versus $31.4(29.8 ; 33.1)$ in females, $=0.15$; for SC-area-2 $43.5(41.4 ; 45.7)$ in males versus $47.6(45.2 ; 50.1)$ in females, $P=0.013)$. After adjustment for differences in weight and height between boys and girls, differences increased and turned significant for measures of preperitoneal fat (geometric mean (CI95\%) for PP-area-2 $28.8(27.5 ; 30.2)$ in males versus 32.4 (30.9; $33.9)$ in females, $P=0.001$; for SC-area- $43.0(40.9 ; 45.0)$ in males versus $48.5(46.0 ; 50.8)$ in females, $P=0.001$, Fig. 4). Both unadjusted and adjusted ratios of preperitoneal to subcutaneous fat did not differ significantly between sexes (geometric mean $(\mathrm{CI} 95 \%)$ for ratio area-2 $0.69(0.65 ; 0.72)$ in males versus $0.66(0.52 ; 0.70)$ in females, $P=0.33)$. All results for PP-area-2 and SC-area-2 are comparable to the other measures of preperitoneal and subcutaneous fat thickness (data not shown).

\section{Effect of age}

During the second year of life we found a pronounced increase of $45 \%$ in preperitoneal fat thickness, while 
Fig. 3 Correlation between fat compartments and body mass index. Correlation between a subcutaneous fat thickness and body mass index and $\mathbf{b}$ preperitoneal fat thickness and body mass index $(n=439)$. **Correlation is significant at the 0.01 level
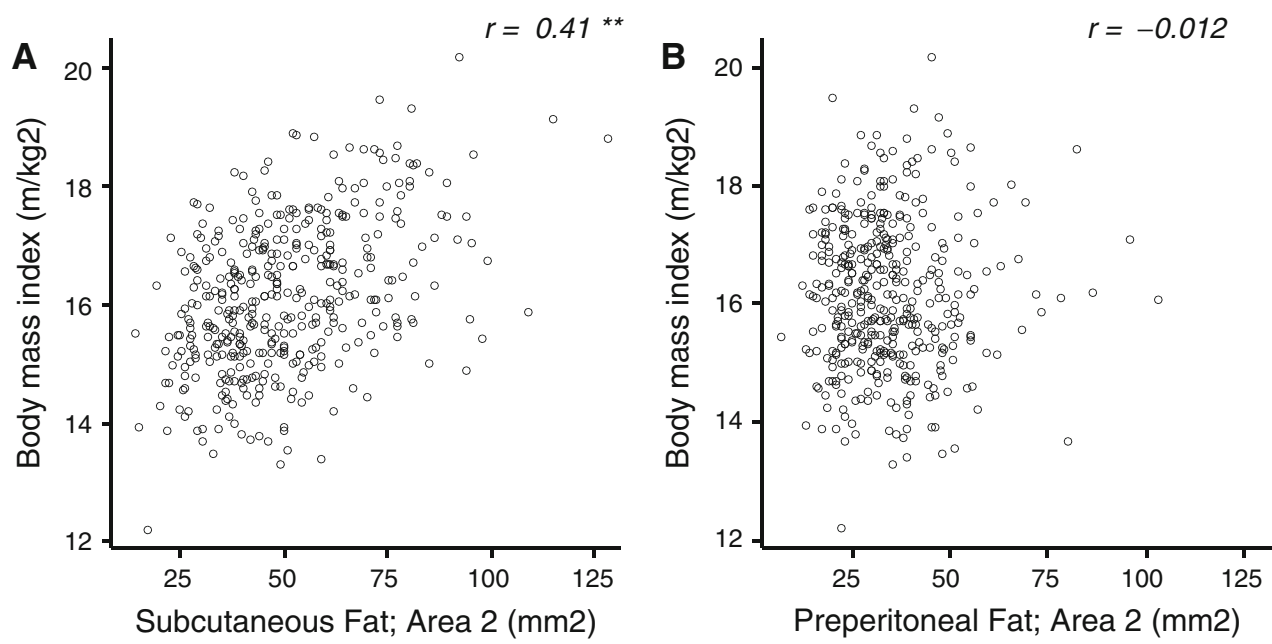

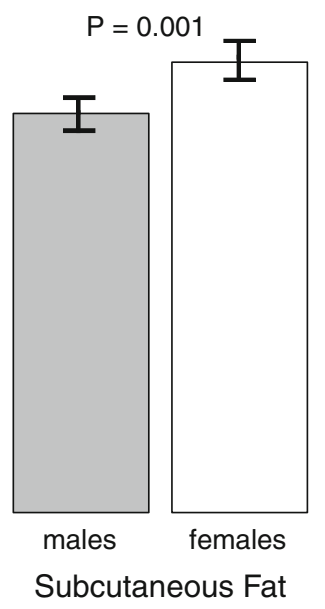

Fig. 4 Effect of sex on abdominal fat compartments. Comparison of preperitoneal and subcutaneous fat thickness in male $(n=210)$ versus female $(n=207)$ infants, adjusted for current height and weight. Bars represent the adjusted geometric mean; error bars represent the $95 \%$ confidence interval of the mean

subcutaneous fat thickness hardly increased [geometric mean (CI95\%) for PP-area-2 $26.7(25.1 ; 28.3)$ at 1 year versus $38.7(37.2 ; 40.3)$ at 2 year, $P<0.001$; for SC-area- 2 $48.5(46.1 ; 51.0)$ at 1 year versus $49.0(46.6 ; 51.3)$ at 2 year, $P=0.78$, Fig. 5]. Consequently, ratios of abdominal fat changed towards a more central fat distribution within the second year of life. This effect was independent of the current BMI of the infants. After adjustment for current weight and height, the increase in preperitoneal fat with age diminished to $27 \%$, while results for subcutaneous fat remained unaltered (geometric mean (CI95\%) for PParea-2 $27.0(25.2 ; 28.9$. $)$ in 1 year versus $34.3(32.1 ; 36.7)$ in 2 year, $P<0.001$; for SC-area-2 $45.2(42.0 ; 48.6)$ in 1 year versus $46.0(42.9 ; 49.4)$ in 2 year, $P=0.78$, Fig. 5$)$.

Comparing the alternative measurements of thickness we found that the difference in preperitoneal fat was largest in the area 2 measurements (Table 3 ). The area 2 measurement

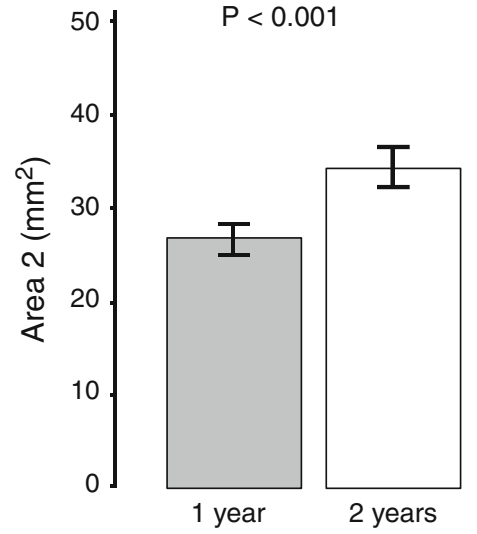

Preperitoneal Fat

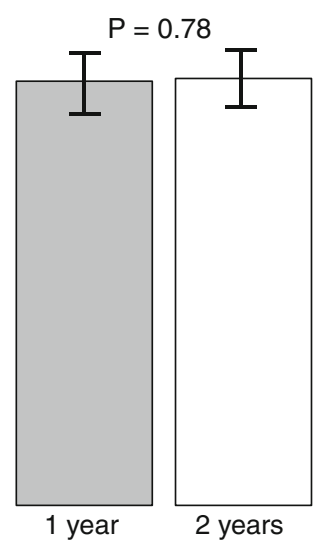

Subcutaneous Fat

Fig. 5 Effect of age on abdominal fat compartments. Comparison of preperitoneal and subcutaneous fat thickness in 1 year old $(n=210)$ versus 2 year old $(n=207)$ infants, adjusted for current height and weight. Bars represent the adjusted geometric mean; error bars represent the $95 \%$ confidence interval of the mean

reflects the developmental change in shape of the preperitoneal fat layer, which transforms from a structure in which the preperitoneal fat layer tapers out at the distal end, to a layer of more constant thickness (Fig. 2).

\section{Discussion}

We describe a sonographic method for quantifying abdominal subcutaneous and preperitoneal fat compartments in infancy. This methodology can be instrumental for investigating abdominal fat development from early life on and will allow examining factors and critical time periods influencing abdominal fat acquisition and distribution in more detail. Most of the data relating to adipose tissue content in infancy are derived from indirect methods [23]. These methods are unable to distinguish between 
abdominal fat components or exclusively estimate subcutaneous fat. Moreover, indirect measures of body composition such as BMI and waist circumference are influenced by confounding factors that differ throughout development, which limit the value of these measures in longitudinal studies.

Our protocol for the sonographic assessment of abdominal fat compartments can be applied to infants with relative ease. As the measurement of preperitoneal and subcutaneous fat distribution has been validated in adults and has already been applied to older children [12, 17] sonographic methods now allow assessment of fat distribution throughout life.

The reproducibility of our method was good to excellent. Potential complications are first that the anatomy of the preperitoneal fat layers is heterogeneous in infants, second that fat layers are very thin in part of the 1 and 2 years old infants and third that the measurements can be distorted by movement artefacts, which complicates the standardization of the technique in infants. Training and experience of the investigators are therefore of critical importance for optimal performance of the technique. We advise to perform joint measurements with all investigators involved to standardize the selection of images for measurements. Measurements should routinely be compared across investigators and over time.

We introduced area measurements and compared them to distance measurements for estimating preperitoneal and subcutaneous fat thickness. Strong correlations between different measurements of the respective fat layer argue that measurements of distance and areas of different length could be used interchangeably. However, ratios of preperitoneal to subcutaneous fat thickness differed considerably (for details, see Table 3) and the increase in preperitoneal fat in the second year of life was largest in area measurement of two centimeter length (PP-area-2). Based on our experience, we interpret these results to reflect age-dependent changes in shape of the preperitoneal fat layer. The increase of preperitoneal fat in the second year of life is also conspicuous if one simply visually inspects the raw sonographic images of the fat layers. The measurement of two-dimensional areas rather than the mere assessment of the one dimensional thickness of fat layer as done in adults by using the abdominal wall fat index [12] captures this increase in preperitoneal fat in the second year of life effectively. A further advantage of area measurements over simple distance measurements is their slightly better reproducibility as found in our study. Based on our results we propose area measurements of $2 \mathrm{~cm}$ length to assess preperitoneal and subcutaneous fat thickness in infancy. To definitely valuate the different sonographic measurements, age specific validation with MRI or CT scan needs to be performed.

Alternative sonographic techniques for estimating visceral fat have been developed in adults [10, 11, 24]. Additionally to the method described here, we assessed visceral fat by measuring the intraabdominal diameter as established by Armellini et al. [10] in the 1 and 2 year old infants (data not shown). In our hands the results of this measurement technique were strongly confounded by factors such as air distension, stooling pattern and compliance of the infants.

To our knowledge direct assessment of age dependent characteristics in abdominal fat distribution have not previously been investigated within the first years of life in a population based prospective study. Only few studies directly assessed abdominal fat compartments and were often restricted to older and special patient groups, primarily obese children and adolescents [25-27]. We observed a substantial increase in preperitoneal fat thickness in the second year of life, whereas subcutaneous fat thickness hardly increased. The observed changes result in a shift in abdominal fat distribution towards more preperitoneal fat during the second year of life. These observations are in line

Table 3 Measures of preperitoneal and subcutaneous fat layers by age and sex

\begin{tabular}{|c|c|c|c|c|}
\hline & \multicolumn{2}{|l|}{1 year } & \multicolumn{2}{|l|}{2 years } \\
\hline & Male $(n=104)$ & Female $(n=108)$ & Male $(n=120)$ & Female $(n=107)$ \\
\hline PP-area-1 (mm²) & $16.0(11.319 .0)$ & $17.0(13.0,21.0)$ & $21.9(17.227 .4)$ & $23.2(19.1,28.8)$ \\
\hline $\mathrm{SC}$-area-1 $\left(\mathrm{mm}^{2}\right)$ & $21.5(17.0,30.0)$ & $24.0(19.0,31.0)$ & $22.0(17.5,29.5)$ & $24.5(19.0,32.5)$ \\
\hline PP-area-2 $\left(\mathrm{mm}^{2}\right)$ & $24.0(20.0,30.8)$ & $28.0(20.0,32.0)$ & $36.9(29.0,45.8)$ & $37.2(30.9,46.4)$ \\
\hline SC-area-2 $\left(\mathrm{mm}^{2}\right)$ & $43.5(33.3,58.8)$ & $49.5(37.0,60.8)$ & $42.3(34.2,57.4)$ & $48.7(38.0,63.0)$ \\
\hline PP-distance $(\mathrm{mm})$ & $2.0(1.6,2.4)$ & $2.0(1.7,2.6)$ & $2.7(2.2,3.1)$ & $2.8(2.2,3.2)$ \\
\hline SC-distance $(\mathrm{mm})$ & $2.0(1.6,2.8)$ & $2.3(1.8,2.9)$ & $2.0(1.6,2.7)$ & $2.4(1.8,2.9)$ \\
\hline Ratio area-1 & $0.7(0.5,0.9)$ & $0.7(0.5,0.8)$ & $1.0(0.8,1.2)$ & $1.0(0.7,1.2)$ \\
\hline Ratio area-2 & $0.6(0.4,0.7)$ & $0.6(0.4,0.7)$ & $0.8(0.7,1.0)$ & $0.8(0.6,1.0)$ \\
\hline Ratio distance & $0.9(0.7,1.2)$ & $0.9(0.7,1.1)$ & $1.2(1.0,1.6)$ & $1.2(1.0,1.5)$ \\
\hline
\end{tabular}

Values are presented as median (25th, 75th percentiles). $P P$ preperitoneal, $S C$ subcutaneous. Ratio $=$ preperitoneal/subcutaneous 
with MRI data showing that in the first months of life approximately $90 \%$ of body fat is located subcutaneously [28]. Longitudinal studies are needed to identify factors influencing abdominal fat acquisition in infancy and investigate whether differences in fat distribution observed in infancy persist throughout childhood.

After adjusting for weight and height, girls had both thicker layers of preperitoneal and subcutaneous fat than boys of the same age. These results are similar to observations in prepubescent children [9], but deviate from findings in adults, where males consistently have larger amounts of visceral fat than females [2, 29, 30, 31]. It has been suggested, that hormonal changes during puberty account for these findings [32]. Furthermore relative fat mass and subcutaneous fat have been reported to be higher in females than males from the first year of life on [2, 29, 30, 33].

Within the first 2 years of life, abdominal subcutaneous fat thickness was only moderately associated with body mass index; preperitoneal fat was virtually unrelated to BMI. As a consequence, the larger the BMI the more the intraabdominal fat ratio shifted towards subcutaneous fat. Similar observations have been reported in studies on fat distribution in adults $[31,34]$. In children results are heterogeneous: Some studies suggested that visceral fat increases in proportion to overall fatness [9], whereas others have shown that obese children tend to accumulate subcutaneous and not visceral fat [30]. Interestingly in small for gestational age newborns, Harrington et al. [35] reported that only subcutaneous fat but not intraabdominal fat was reduced. The relation of BMI with preperitoneal fat amount and fat distribution may differ throughout growth. Long-term observations have to address age and weight dependent effects in more detail. Beyond, these results suggest that for clinical use it may be misleading to focus on fat distribution rather than on total amounts of fat, if BMI as a parameter of obesity is not taken into account.

Limitations of our study include that we did not measure the total amount of intraabdominal fat, but quantified preperitoneal fat layer as an estimate for intraabdominal or visceral fat. So far, data are lacking to what extent preperitoneal fat correlates with quantities of visceral fat in infants and how stable this association is throughout life. Suzuki et al. showed that the abdominal wall fat index is strongly correlated with a corresponding ratio of visceral to subcutaneous fat measured by CT scan in adults [12]. Moreover, in both, children and adults, preperitoneal fat has been shown to be associated with metabolic and cardiovascular risk factors or disease [11, 16, 17, 34, 36]. The present study was restricted to infants of Dutch ethnicity and cannot be generalized to other ethnic groups, since ethnicity has been shown to be associated with body fat distribution [37, 38]. The study population consisted of healthy infants in the normal BMI range, obesity as well as low birth weight or prematurity were rare.

Here we describe a protocol for the sonographic assessment of central subcutaneous and preperitoneal fat. We show that the method can be applied-with a high degree of reproducibility-for investigating the regional fat distribution in 1 and 2 year old infants. We therefore expect that this methodology will help elucidating how fat distribution in early life determines risk factors for associated disease.

Acknowledgments The first phase of the Generation R study is made possible by financial support from the Erasmus Medical Center Rotterdam, the Erasmus University Rotterdam and the Netherlands Organization for Health Research and Development (ZonMw). The study described here was supported by an unrestricted grant from Numico Research. We are very thankful to all of the children and parents who took part in the study. We gratefully acknowledge the contribution of general practitioners, hospitals, midwives and pharmacies in Rotterdam and would like to thank Miranda Geelhoed and Bero Verburg for their help in early parts of the study.

Open Access This article is distributed under the terms of the Creative Commons Attribution Noncommercial License which permits any noncommercial use, distribution, and reproduction in any medium, provided the original author(s) and source are credited.

\section{References}

1. Kopelman PG. Obesity as a medical problem. Nature. 2000;404 (6778):635-43.

2. Fox CS, Massaro JM, Hoffmann U, Pou KM, Maurovich-Horvat $\mathrm{P}$, Liu CY, et al. Abdominal visceral and subcutaneous adipose tissue compartments: association with metabolic risk factors in the framingham heart study. Circulation. 2007;116(1):39-48. doi: 10.1161/CIRCULATIONAHA.106.675355.

3. Fujimoto WY, Bergstrom RW, Boyko EJ, Chen KW, Leonetti DL, Newell-Morris L, et al. Visceral adiposity and incident coronary heart disease in Japanese-American men. The 10-year follow-up results of the Seattle Japanese-American Community Diabetes Study. Diabetes Care. 1999;22(11):1808-12. doi:10.2337/diacare. 22.11.1808.

4. Goran MI, Gower BA. Relation between visceral fat and disease risk in children and adolescents. Am. J. Clin. Nutr. 1999;70(1): 149S-56S.

5. Owens S, Gutin B, Ferguson M, Allison J, Karp W, Le NA. Visceral adipose tissue and cardiovascular risk factors in obese children. J. Pediatr. 1998;133(1):41-5. doi:10.1016/S0022-3476 (98)70175-1.

6. Rossner S, Bo WJ, Hiltbrandt E, Hinson W, Karstaedt N, Santago $\mathrm{P}$, et al. Adipose tissue determinations in cadavers-a comparison between cross-sectional planimetry and computed tomography. Int. J. Obes. 1990;14(10):893-902.

7. Sobol W, Rossner S, Hinson B, Hiltbrandt E, Karstaedt N, Santago $\mathrm{P}$, et al. Evaluation of a new magnetic resonance imaging method for quantitating adipose tissue areas. Int. J. Obes. 1991; 15(9):589-99.

8. Wells JC. The programming effects of early growth. Early Hum. Dev.. 2007;83(12):743-8.

9. Goran MI. Visceral fat in prepubertal children: influence of obesity, anthropometry, ethnicity, gender, diet, and growth. Am. 
J. Hum. Biol. 1999;11(2):201-7. doi:10.1002/(SICI)1520-6300 (1999) 11:2<201::AID-AJHB8>3.0.CO;2-R.

10. Armellini F, Zamboni M, Rigo L, Robbi R, Todesco T, Castelli S, et al. Measurements of intra-abdominal fat by ultrasound and computed tomography: predictive equations in women. Basic. Life. Sci. 1993;60:75-7.

11. Liu KH, Chan YL, Chan WB, Kong WL, Kong MO, Chan JC. Sonographic measurement of mesenteric fat thickness is a good correlate with cardiovascular risk factors: comparison with subcutaneous and preperitoneal fat thickness, magnetic resonance imaging and anthropometric indexes. Int. J. Obes. Relat. Metab. Disord. 2003;27(10):1267-73. doi:10.1038/sj.ijo.0802398.

12. Suzuki R, Watanabe S, Hirai Y, Akiyama K, Nishide T, Matsushima $\mathrm{Y}$, et al. Abdominal wall fat index, estimated by ultrasonography, for assessment of the ratio of visceral fat to subcutaneous fat in the abdomen. Am. J. Med. 1993;95(3):309-14. doi:10.1016/ 0002-9343(93)90284-V.

13. Yamamoto M, Egusa G, Hara H, Yamakido M. Association of intraabdominal fat and carotid atherosclerosis in non-obese middle-aged men with normal glucose tolerance. Int. J. Obes. Relat. Metab. Disord. 1997;21(10):948-51. doi:10.1038/sj.ijo.0800501.

14. Yamamoto M, Egusa G, Yamakido M. Carotid atherosclerosis and serum lipoprotein(a) concentrations in patients with NIDDM. Diabetes. Care. 1997;20(5):829-31. doi:10.2337/diacare.20.5.829.

15. Soyama A, Nishikawa T, Ishizuka T, Ito H, Saito J, Yagi K, et al. Clinical usefulness of the thickness of preperitoneal and subcutaneous fat layer in the abdomen estimated by ultrasonography for diagnosing abdominal obesity in each type of impaired glucose tolerance in man. Endocr. J. 2005;52(2):229-36. doi:10.1507/ endocrj.52.229.

16. Merino-Ibarra E, Artieda M, Cenarro A, Goicoechea J, Calvo L, Guallar A, et al. Ultrasonography for the evaluation of visceral fat and the metabolic syndrome. Metabolism. 2005;54(9):1230-5. doi:10.1016/j.metabol.2005.04.009.

17. Tanaka Y, Kikuchi T, Nagasaki K, Hiura M, Ogawa Y, Uchiyama M. Lower birth weight and visceral fat accumulation are related to hyperinsulinemia and insulin resistance in obese Japanese children. Hypertens. Res. 2005;28(6):529-36. doi:10.1291/ hypres.28.529.

18. Jaddoe VW, Mackenbach JP, Moll HA, Steegers EA, Tiemeier H, Verhulst FC, et al. The generation R study: design and cohort profile. Eur. J. Epidemiol. 2006;21(6):475-84.

19. Hofman A, Jaddoe VW, Mackenbach JP, Moll HA, Snijders RF, Steegers EA, et al. Growth, development and health from early fetal life until young adulthood: the generation R study. Paediatr. Perinat. Epidemiol. 2004;18(1):61-72. doi:10.1111/j.1365-3016. 2003.00521.x.

20. Fredriks AM, van Buuren S, Burgmeijer RJ, Meulmeester JF, Beuker RJ, Brugman E, et al. Continuing positive secular growth change in The Netherlands 1955-1997. Pediatr. Res. 2000;47(3): 316-23. doi:10.1203/00006450-200003000-00006.

21. Fredriks AM, van Buuren S, Wit JM, Verloove-Vanhorick SP. Body index measurements in 1996-7 compared with 1980. Arch. Dis. Child. 2000;82(2):107-12. doi:10.1136/adc.82.2.107.

22. Barlow SE, Dietz WH. Obesity evaluation and treatment: expert committee recommendations. The maternal and child health bureau, health resources and services administration and the department of health and human services. Pediatrics. 1998;102 (3):E29. doi:10.1542/peds.102.3.e29.

23. Wells CJ. The programming effects of early growth. Early Hum. Dev. 2007;83(12):743-8.
24. Stolk RP, Meijer R, Mali WP, Grobbee DE, van der Graaf Y. Ultrasound measurements of intraabdominal fat estimate the metabolic syndrome better than do measurements of waist circumference. Am. J. Clin. Nutr. 2003;77(4):857-60.

25. Nishina M, Kikuchi T, Yamazaki H, Kameda K, Hiura M, Uchiyama M. Relationship among systolic blood pressure, serum insulin and leptin, and visceral fat accumulation in obese children. Hypertens. Res. 2003;26(4):281-8. doi:10.1291/hypres.26.281.

26. Asayama K, Dobashi K, Hayashibe H, Kodera K, Uchida N, Nakane T, et al. Threshold values of visceral fat measures and their anthropometric alternatives for metabolic derangement in Japanese obese boys. Int. J. Obes. Relat. Metab. Disord. 2002;26 (2):208-13. doi:10.1038/sj.ijo.0801865.

27. Semiz S, Ozgoren E, Sabir N. Comparison of ultrasonographic and anthropometric methods to assess body fat in childhood obesity. Int. J. Obes. 2007;31(1):53-8.

28. Olhager E, Flinke E, Hannerstad U, Forsum E. Studies on human body composition during the first 4 months of life using magnetic resonance imaging and isotope dilution. Pediatr. Res. 2003;54(6): 906-12. doi:10.1203/01.PDR.0000088064.63106.5E.

30. Kuk JL, Lee S, Heymsfield SB, Ross R. Waist circumference and abdominal adipose tissue distribution: influence of age and sex. Am. J. Clin. Nutr. 2005;81(6):1330-4.

31. Fox K, Peters D, Armstrong N, Sharpe P, Bell M. Abdominal fat deposition in 11-year-old children. Int. J. Obes. Relat. Metab. Disord. 1993;17(1):11-6.

32. Lemieux S, Prud'homme D, Bouchard C, Tremblay A, Despres JP. Sex differences in the relation of visceral adipose tissue accumulation to total body fatness. Am. J. Clin. Nutr. 1993;58 (4):463-7.

33. de Ridder CM, Thijssen JH, Bruning PF, Van den Brande JL, Zonderland ML, Erich WB. Body fat mass, body fat distribution, and pubertal development: a longitudinal study of physical and hormonal sexual maturation of girls. J. Clin. Endocrinol. Metab. 1992;75(2):442-6. doi:10.1210/jc.75.2.442.

34. Butte NF, Hopkinson JM, Wong WW, Smith EO, Ellis KJ. Body composition during the first 2 years of life: an updated reference. Pediatr. Res. 2000;47(5):578-85. doi:10.1203/00006450-20000 5000-00004.

35. Minocci A, Guzzaloni G, Marzullo P, Savia G, Tagliaferri M, Berselli ME, et al. Abdominal fat index by ultrasound does not estimate the metabolic risk factors of cardiovascular disease better than waist circumference in severe obesity. Diabetes. Metab. 2005; 31(5):471-7. doi:10.1016/S1262-3636(07)70218-5.

36. Harrington TA, Thomas EL, Frost G, Modi N, Bell JD. Distribution of adipose tissue in the newborn. Pediatr. Res. 2004;55 (3):437-41. doi:10.1203/01.PDR.0000111202.29433.2D.

37. Liu KH, Chan YL, Chan WB, Chan JC, Chu CW. Mesenteric fat thickness is an independent determinant of metabolic syndrome and identifies subjects with increased carotid intima-media thickness. Diabetes. Care. 2006;29(2):379-84. doi:10.2337/diacare.29. 02.06.dc05-1578.

38. Liska D, Dufour S, Zern TL, Taksali S, Cali AM, Dziura J, et al. Interethnic differences in muscle, liver and abdominal fat partitioning in obese adolescents. PLoS. ONE. 2007;2(6):e569. doi: 10.1371/journal.pone.0000569.

39. Lear SA, Humphries KH, Kohli S, Chockalingam A, Frohlich JJ, Birmingham CL. Visceral adipose tissue accumulation differs according to ethnic background: results of the Multicultural Community Health Assessment Trial (M-CHAT). Am. J. Clin. Nutr. 2007;86(2):353-9. 